\title{
Stage IIIA Appendix Carcinoma AJCC v7
}

National Cancer Institute

\section{Source}

National Cancer Institute. Stage IIIA Appendix Carcinoma A/CC v7. NCI Thesaurus. Code C87803.

Stage IIIA includes: (T1, N1, M0); (T2, N1, M0). T1: T umor invades submucosa. T2:

Tumor invades muscularis propria. N1: Metastasis in 1-3 regional lymph nodes. M0: No distant metastasis. (AJCC 7th ed.) 